\title{
Fabrication and Characterization of Magnetic Nanostructures with Different Iron Concentration.
}

\author{
M. Takeguchi", M. Shimojo ${ }^{* * *}$, R. Che and K. Furuya ${ }^{*}$ \\ *High Voltage Electron Microscopy Station, National Institute for Materials Science, \\ 3-13 Sakura, Tsukuba, 305-0003, Japan \\ ** Advanced Science Research Laboratory, Saitama Institute of Technology, \\ 1690 Fusaiji, Fukaya, 369-0293, Japan
}

Electron beam induced deposition (EBID) has been becoming one of the most promising methods in point of view of finely high resolution, abundant types of deposit elements, and capability of three dimensional fabrications. Recently, EBID with precursors containing magnetic elements such as iron and cobalt has been employed to fabricate ferromagnetic nanostructures [1-3]. In EBID, metal-organic gases are decomposed by electron beams, resulting in the deposition of metals at the desired positions. The deposits contain a considerable amount of carbon in addition to metal element [4-6]. This unfavorable carbon comes from ligands of the dissociated organic precursors, and to control the carbon concentration has been a significant issue, because the compositional ratio of metal to carbon in EBID nanostructures significantly influences electronic and magnetic properties. In the present work, dual gas-introduction pipelines were installed into a scanning electron microscope (SEM), and nanostructures with the different compositional ratio of iron to carbon were formed using a mixture gas of iron carbonyl and ferrocene. Using this dual gas EBID system, the iron to carbon compositional ratio in the EBID nanostructures was successfully controlled by changing the partial pressure of these gases. The iron and carbon concentration in the nanostructures was measured by energy dispersive X-ray spectrometer (EDS) and their magnetic properties were evaluated by electron holography.

A field emission gun SEM, JSM-7800F-UHV, was employed to carry out EBID experiments. A gas introduction system combined with dual gas source reservoirs and pipelines through leak valves was installed into the SEM so that the partial pressure of two kinds of gases could be independently controlled and their mixture could be introduced into a specimen chamber of the SEM via a nozzle with a inner diameter about $0.2 \mathrm{~mm}$. Transmission electron microscopy (TEM) observation and off-axis electron holography were performed in a $300 \mathrm{kV}$ field emission gun transmission electron microscope, JEOL JEM-3000F, equipped with an electron biprism. The objective lens was turned off during electron holography experiment and a mini objective lens located below the objective lens was used to form off-axis holograms on a Gatan ultrascan CCD camera integrated in a Gatan imaging filter.

Figure 1a shows a SEM image of nanorods (numbered from 1 to 10) formed by EBID with various partial pressures of iron carbonyl and ferrocene gases on an edge of a molybdenum thinned area. First, the partial pressure of ferrocene gas was maintained at $1 \times 10^{-5} \mathrm{~Pa}$. Then, carbonyl gas was introduced so that the total pressure was increased from $3 \times 10^{-5} \mathrm{~Pa}$ to $12 \times 10^{-5} \mathrm{~Pa}$ by $1 \times 10^{-5} \mathrm{~Pa}$ for each EBID fabrication of nanorod (totally 10 nanorods were formed). Figure $1 \mathrm{~b}$ shows the ratio of the partial pressure of carbonyl 
$\left(\mathrm{P}_{\mathrm{c}}\right)$ to the sum of $\mathrm{P}_{\mathrm{c}}$ and the partial pressure of ferrocene $\left(\mathrm{P}_{\mathrm{f}}\right)$ for each nanorod. Figure 2 shows iron-L peak intensity for each nanorod acquired by EDS analysis, indicating that the iron concentration was successfully increased depending on the change of $\mathrm{P}_{\mathrm{c}} /\left(\mathrm{P}_{\mathrm{c}}+\mathrm{P}_{\mathrm{f}}\right)$.

The remanent magnetic flux density $B_{r}$ for each nanorod was evaluated by off-axis electron holography. Figure $3 \mathrm{a}$ and $3 \mathrm{~b}$ show an electron hologram of the No.3 to No.6 nanorods and the corresponding reconstructed phase image, respectively. The detailed phase image analyses indicated that the remanent magnetic flux density $B_{r}$ of the EBID nanostructures containing iron and carbon is related to the iron concentration.

\section{References}

[1] R. R. Kunz and T. M. Mayer, Appl. Phys. Lett. 50 (1987) 962.

[2] I. Utke et al., Appl. Phys. Lett. 80 (2002) 4792.

[3] M. Takeguchi et al., Nanotechnology 16 (2005) 1321.

[4] M. Han et al., Philos. Mag. 84 (2004) 1281.

[5] M. Shimojo et al., Appl. Phys. A 79 (2004) 1869.

[6] M. Takeguchi et al., Jpn. J. Appl. Phys. 44 (2005) 5631.
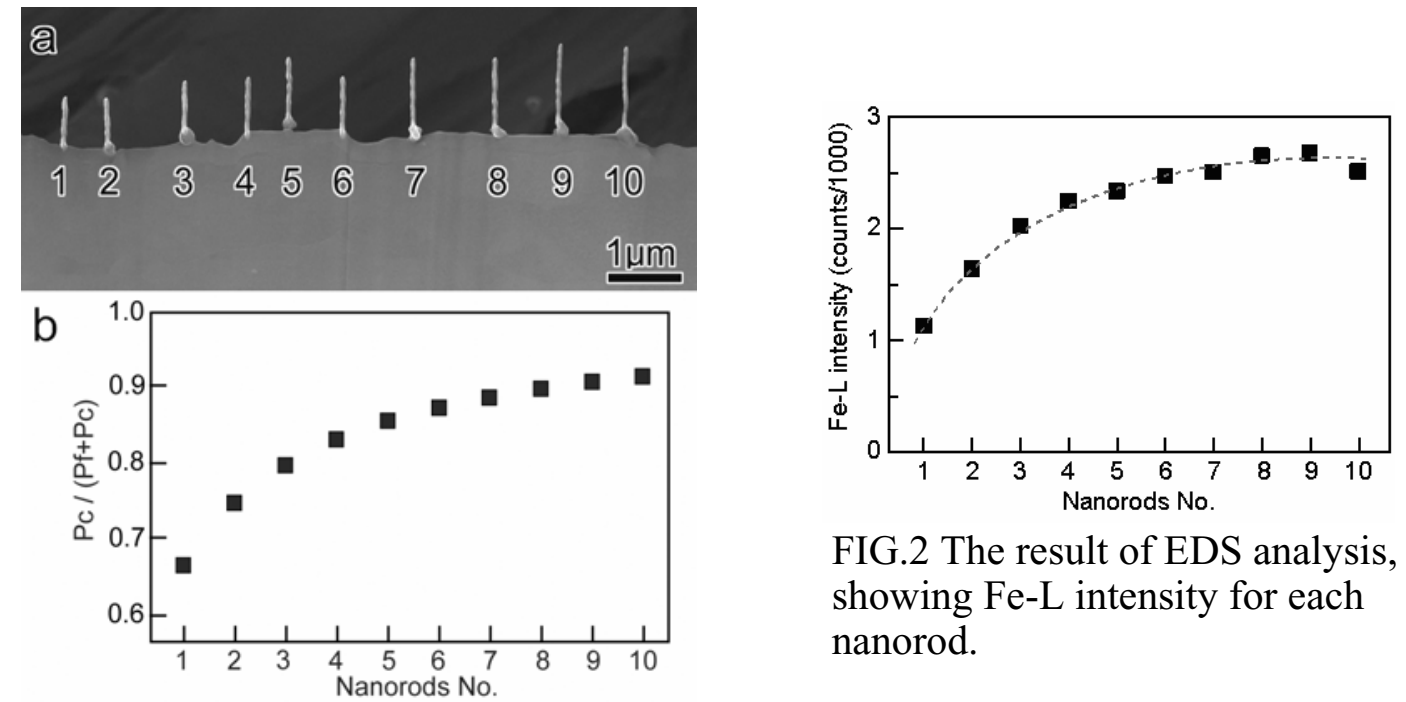

FIG.2 The result of EDS analysis, showing Fe-L intensity for each nanorod.

FIG.1 (a) SEM image of the EBID fabricated nanorods. (b) The ratio of the partial pressure of carbonyl $\left(\mathrm{P}_{\mathrm{c}}\right)$ to the sum of $\mathrm{P}_{\mathrm{c}}$ and the partial pressure of ferrocene $\left(\mathrm{P}_{\mathrm{f}}\right)$ for each nanorod.

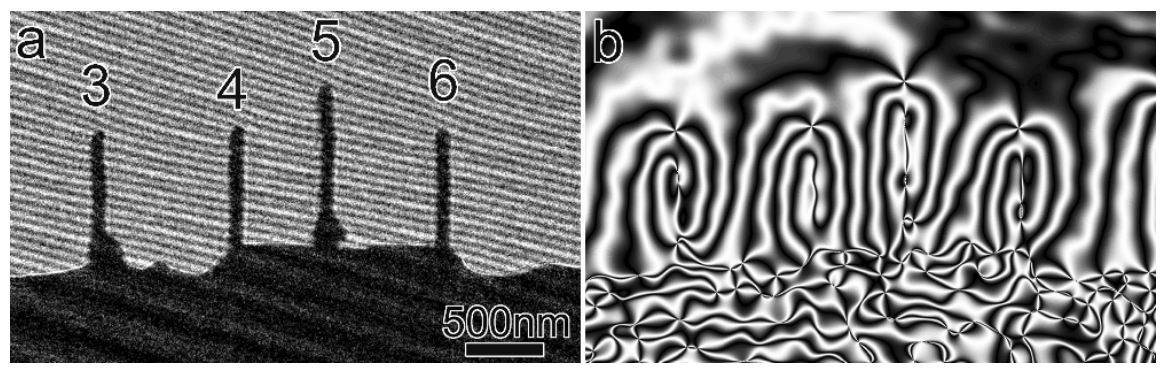

FIG.3 (a) Electron hologram of the No.3 6 nanorods. (b) Phase image (interference micrograph) reconstructed from (a) with an amplification factor of 4. 